\title{
COMPUTER-ASSISTED KNEE TOTAL ARTHROPLASTY
}

\author{
Roberto Freire da Mota e Albuquerque ${ }^{1}$, fábio Jansen Angelin², José Ricardo Pécora ${ }^{1}$, \\ Marco Martins AmatuzZi ${ }^{3}$, Sandra Umeda Sasak ${ }^{4}$
}

\section{SUMMARY}

One of the most significant technological advancements in current medicine is the computer-assisted surgery, which, for orthopaedics, one of the major uses of this technology is in knee arthroplasty. The main contribution provided by computer-assisted orthopaedic surgery (CAOS) to knee arthroplasty is its potential to improve prosthesis implant precision and the operated limb alignment, contributing to results optimization and longevity. The image-independent navigation, based on anatomical references acquired during surgical procedure through infrared rays' transmitters, has been the prevailing technique in knee arthroplasty. We used the navigation system "OrthoPilot" version 2.2 in total knee arthroplasty for inserting
72 knee prosthesis "Search Evolution" by "Aesculap" with or without posterior stabilization in a continuous series. The objective was to measure the accuracy of the alignment achieved with navigation through wide-angled X-ray images obtained postoperatively. We found a mean deviation for the null mechanical axis of $0.66^{\circ}$ with standard deviation of $0.7^{\circ}$, with $98.6 \%$ of the knees being within an error margin of $3^{\circ}$ and $78.2 \%$ with error lower than $1^{\circ}$. We concluded that the system is safe and accurate, not causing additional morbidity to conventional surgery.

Keywords: Knee; Arthroplasty, Replacement, Knee; Knee Prosthesis; Surgery, Computer-Assisted.

\section{INTRODUCTION}

Technological evolution has marked the recent history of medicine, revolutionizing diagnostic and therapeutic methods in all its fields. In this context, one of the most recent achievements is the computer-aided orthopaedic surgery (CAOS). The development and clinical application of computer-assisted surgery systems in orthopaedics has recently resulted in the establishment of a specific international society, the CAOS - International, the first meeting of which occurred in 2001, in Davos, Switzerland. In our environment, we got the first computed-assisted orthopaedic surgery system in 1999, at the Orthopaedics and Traumatology Institute, Hospital das Clínicas, University of São Paulo, which was donated by Aesculap Academy. That system, named OrthoPilot, is intended to Surgical Navigation in Orthopaedics, its major application is on Knee Total Arthroplasty, with which we had our first experience in this area. OrthoPilot was the first navigation system for knee arthroplasty to be widely employed in clinics, especially in Europe. Navigation consists of surgical instrumentation guidance, offering objective intra-operative parameters to surgeons, providing accuracy and safety to a surgical procedure, enabling the performance of minimally invasive procedures, and reducing or mitigating exposure to $X$-ray. Thus, navigation is the surgical or intra-operative arm of CAOS, which can also be applied in surgical planning, results simulation, and robotics. Many reference possibilities exist for navigation systems, those based on images that can be captured before or during surgical procedure (computed tomography (CT), magnetic resonance (MR), fluoroscopy, ultra-sound (US), etc) and those that are not based on images, but in anatomical references captured during surgical procedure. OrthoPilot fits in the latter group, with particular anatomical points of a patient are sent to the computer during surgery through infrared (IR) active conveyors, which are found by an optical tracking system similar to a GPS (Figure 1). The position and movement of those points are followed up in real time by the computer, allowing to find the core of the three major joints of a patient's lower limb by a mathematic algorithm applied to the performance of standardized movements of those joints, thus enabling a virtual reconstruction of the lower limb's mechanical axis. The association of this lower limb virtual model to anatomical references achieved ant to the presence of IR conveyors (Figure 2) in tibial and femoral section guides enable bone sections to be performed in a surgical procedure to be guided by the computer in an accuracy degree equal or lower than $1^{\circ}$. The system "sees" the section guides and their position towards tibial and femoral joint surface and to lower limb's mechanical axis, providing a feedback to the surgeon by means of a virtual image on screen with objective parameters about the result to be achieved as a result of section guides position, namely: section thickness and angle towards mechanical axis at frontal and sagittal planes (Figure 3). Therefore, conventional alignment guides are not used, with intra- or extra-medullar reference, thus reducing the risk of thromboembolic complications as well. Here, we report the results of lower limbs alignment for our first series of 72 knees in 68 patients submitted

Study developed at the Orthopaedics and Traumatology Institute, Hospital das Clínicas, Medical College, University of São Paulo.

Correspondences to: Av. Nove de Julho, 5.519 cj.41 - São Paulo, SP - CEP 01407-200 - E-mail: rmotaa@uol.com.br

1 - PhD, Orthopaedics and Traumatology Institute, Hospital das Clínicas, Medical College, University of São Paulo.

2 - Assistant Doctor, Orthopaedics and Traumatology Institute, Hospital das Clínicas, Medical College, University of São Paulo.

3 - Emeritus Professor, Department of Orthopaedics and Traumatology, Medical College, University of São Paulo.

4 - Post-graduation student, Orthopaedics and Traumatology Institute, Hospital das Clínicas, Medical College, University of São Paulo.

Received in: 03/30/06; approved in: 05/28/06 
to knee total arthroplasty guided by that navigation system, who were operated between November 200 and March 2002. Our first objective was to check system's accuracy regarding mechanical axis alignment at frontal plane.

\section{MATERIALS AND METHODS}

Seventy two knees of 68 patients assigned for primary knee total arthroplasty (KTA) were sequentially operated. Forty nine patients were females and 19 were males, and mean age was 61.8 years old, ranging from 26 to 81 years old. Cases to be operated with the navigation system were randomly assigned, being included all cases assigned for KTA, regardless of etiology, degree of deformity, or any other variable. From 72 knees, 52 were patients diagnosed with arthrosis and 20 with rheumatoid arthritis. The preoperative mean deviation of axis was $9.25^{\circ}$, with standard deviation of $5.2^{\circ}$, ranging from $17.3^{\circ}$ varus to $22.5^{\circ}$ valgus, always towards lower limb's mechanical axis.

Implants Aesculap model "Search Classic" with or without posterior stabilization, according to each case's indication. Surgeries were all "navigated" with OrthoPilot system (Figure 3) with software release 2.2 for knee total arthroplasty, using 3 modified Schanz pins for attaching IR conveyors, one at the distal femur and one at proximal tibia, which are introduced by an usual arthroplasty incision, and a third percutaneous screw at iliac crest as a reference for finding hip center through the dynamic maneuvers standardized by system. Software is fed with dynamic information for the 3 joints of operated lower limb by means of standardized movements requested by system and by mans of static anatomical references, namely: deepest point of the most compromised tibial plateau, joint surface center of the tibia, femoral distal end, femoral anterior cortical proximally to joint surface, ankle malleolus apex, and ankle anterior joint interline center. Those points are intended to allow software calculations of the femoral component size, sections thickness compared to joint surfaces, and matching anatomical references with dynamic references as a way to measure and increase the reliability of joint center calculations. Femoral distal section and tibial proximal section have been guided by OrthoPilot targeting the null alignment of the mechanical axis, superposing

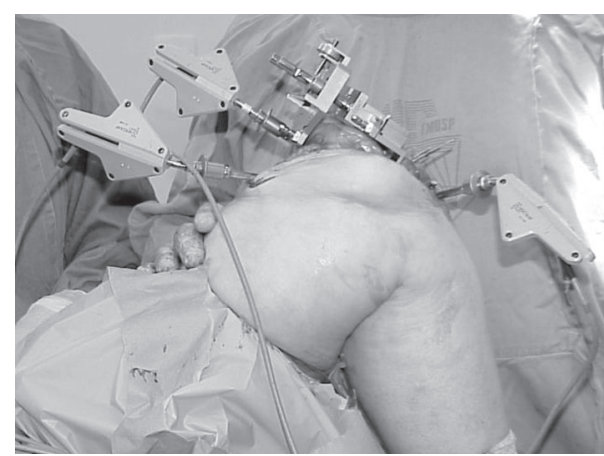

Figure 1 - Rigid bodies with infrared conveyors attached to distal femur, proximal tibia, and femoral section guide.

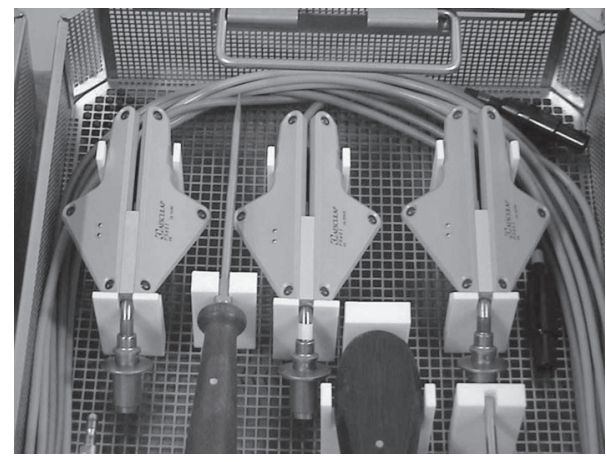

Figure 2 - Rigid bodies with active infrared conveyors for navigation with OrthoPilot.

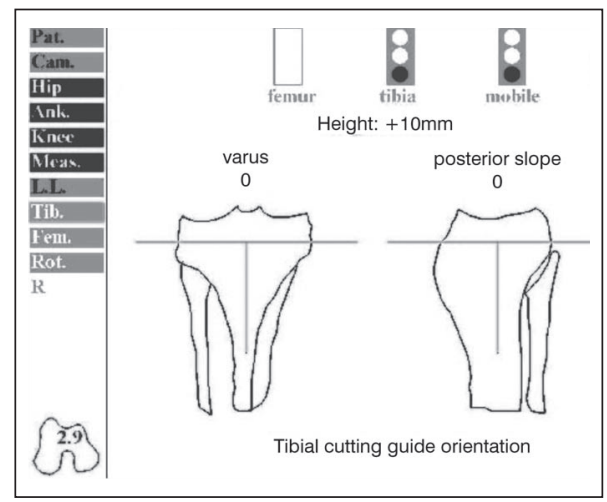

Figure 3 - Screen image with illustration and numeric reference of the tibial section guide positioning. hip's center, knee and ankle in a straight line. The other sections were made with conventional section guides, specific to selected implant and regardless of limb alignment. Joint balance was controlled by the OrthoPilot, which enables detecting any con surgery is continuous, allowing for deviations, thus the procedure is navigated up to implantation and final cementation of components, when final alignment system's database.

perative and postoperative mechanical axis was meay means of full-range $X$-ray images of lower limbs, performed up to 1 month prior to surgery and as soon as the patient was able to remain in an orthostatic position at postoperative period (Figure 4).

\section{RESULTS}

All patients were followed up during one year after surgery, the alignment results were obtained at early postoperative period, once the objective of this study was to measure system's accuracy in obtaining a desired alignment. Within that period, no patient presented with deep venous thrombosis or thromboembolic incidents. One patient evolved with a superficial infection, which responded to oral antibiotic therapy, and one patient presented with a deep infection requiring prosthesis removal and spacer insertion for treating that infection. There were no complications associated to the use of the navigator, or to the insertion of modified Schanz pins.

The average deviation of the null mechanical axis was $0.66^{\circ}$ with standard deviation of $0.7^{\circ}$, with $98.6 \%$ of the knees being within an error margin of $3^{\circ}$, and $79.2 \%$ within an error below $1^{\circ}$. Error ranged from $3.4^{\circ}$ varus to $2.7^{\circ}$ valgus. If we analyze the first 20 knees, of 19 patients, the average deviation was $0.72^{\circ}$ with standard deviation of $0.96^{\circ}, 95 \%$ of the cases with an error below $3^{\circ}$ and $80 \%$ below $1^{\circ}$. All cases with deviation above $3^{\circ}$ have occurred in this early series. At the other end, the last 20 knees of the series presented with an average axis deviation of $0.52^{\circ}$ with standard deviation of $0.68^{\circ}, 100 \%$ of the cases within $3^{\circ}$ margin, and $80 \%$ with deviation below $1^{\circ}$ (Table 1). 


\section{DISCUSSION}

Knee total arthroplasty is a highly complex surgical procedure requiring extensive training for the surgeon and having a long learning curve. Furthermore, its results and implants longevity are closely related to the accuracy and technical strictness of its insertion $(1,2,3,4)$. The proper alignment of femoral and tibial components to lower limb's mechanical axis are certainly one of the most important factors for an implant duration over time, and many studies indicate that a deviation above $3^{\circ}$ implies in premature failure and inferior functional outcomes (1,3,5-9). It is estimated that about $10 \%$ of knee arthroplasties present an alignment error above $3^{\circ}$ even in the hands of experienced surgeons using modern mechanical alignment guides (9). It is evident that other variables are involved, such as tension balancing for peripheral ligaments as well as for posterior cruciate ligament when this is saved, the design and quality of the prosthetic components themselves, and the maintenance of joint interline height, among others ${ }^{(10)}$.

The computer-assisted insertion of knee prosthesis enables the possibility of controlling technical variables of insertion with accuracy and reproducibility controlled by objective, measurable, and intraoperatively documented parameters. This technology brings the expectation of increasing implants longevity by increasing the precision of its alignment and ligamentar balancing ${ }^{(3,10-17)}$, shortening the learning curve for surgeons, and providing more homogeneous results, which are less dependent on each individual's skills. Additionally, it will be an extremely valuable tool for minimally-invasive surgeries development, where the surgeon has a narrower view of the surgical field, increasing the risks of technical inaccuracies. Additionally, the mitigation of intramedullar alignment guides reduced the incidence of thromboembolism (18) and reduces intra-operative blood loss ${ }^{(19)}$. We are still in the early phases of computer-assisted surgery systems development, and many expected benefits are certainly to come, with the improvement and evolution of current systems and softwares. The OrthoPilot's software release 2.2 used in this series provides us with femoral and tibial components alignment control with an accuracy of less than one degree, helps on balancing ligaments and on determining the height of femoral distal and tibial proximal section. Our first objective is to verify the prosthesis alignment accuracy at frontal plane.

OrthoPilot is the navigation system for KTA with the highest number of clinical cases and with the largest series published on international literature ${ }^{(11,17)}$, with this series being the first one in our environment. In international literature, the first series - with 30 knees - was published in 2001 by Mielke et al. ${ }^{(20)}$. Therefore, we started our experience before that first publication. We closed our series when our system was updated with the software release 3.0, in which time point a new series started and shall be reported in the near future. The technology used - not dependent on imaging tests - guided by intra-operative IR conveyors has resulted in a simple, fast, surgeon-friend system, regardless surgeons' familiarity with computers. This is a tool that helps surgeons to perform the procedure with objective parameters and data, with no interference on surgical planning or strategy.

The results achieved are similar to or outperform the alignment of the most accurate series published in literature. One of computer-assisted KTA's differentials is the reduction or mitigation of cases "outlining" from the safety zone of $3^{\circ}(16,17)$. Thus, in our series, we noticed that although the number of cases within an optimal zone of $1^{\circ}$ of accuracy remains almost unchanged in $80 \%$ whether in the first 20 knees, or the whole series, or in the last 20 knees, all "outliner" cases have occurred among the first 20 knees of the series. Therefore, we saw a fast learning curve and the virtual mitigation of safety zone "outliners" after this initial phase.

The presence of a continuous real-time control for limb alignment without the need of mounting visual measurement instruments enables "finding" errors and inaccuracies caused by some maneuvers and procedures during surgery and providing us knowledge that can be transferred to conventional surgeries. We noticed that section guides fixation with nails introduced with the aid of hammers yield significant orientation deviations, which are minimized with the use of threaded pins inserted with perforating drills. Here is another advantage of the navigator: any deviation of section guides positioning during its handling and fixation, or after their support system removal after fixation is immediately detected, not requiring any additional step. Usually, we make use of spacers and/ or tensors to assess flexion and extension spaces as well as ligaments balance during surgery, and here we notice another source of loss of initial alignment, especially in those cases where bone tissue is less stiff, as in patients with rheumatic diseases. We could see that, in some cases, the introduction of a spacer at test and balancing phase may lead to compression on the tensest compartment of the joint, deforming initial section surface and leading to a deviation on final alignment if not corrected.

Navigation in knee arthroplasty has certainly a lot to im- 
prove; newer versions already have control - and should improve it further - on ligaments balancing, components' rotation and dimensioning, extensor apparatus alignment, and the development of instruments that better fits to minimally invasive surgery ${ }^{(21-23)}$. The latter is one of the most promising and significant applications of the navigation, once the reduced visual control in minimally invasive surgeries may be offset by virtual control obtained with navigation (22).

We believe we are just in the early days of a time when computer-assisted surgery will be an essential and usual tool for surgeons as new techniques and instruments are developed, promoting a quality leap in all surgical areas.

\section{CONCLUSIONS}

- Navigation of knee total arthroplasty with OrthoPilot release 2.2 is precise, providing accurate and reproducible alignment of operated limbs.

- Navigation with OrthoPilot release 2.2 is safe, not adding morbidity to conventional surgery.

\section{REFERENCES}

1. Dorr LD, Boiardo RA. Technical considerations in total knee arthroplasty. Clin Orthop. 1997; 205:5-11.

2. Teter KE, Bregman D, Colwell CW Jr. Accuracy of intramedullary versus extramedullary tibial alignment cutting systems in total knee arthroplasty. Clin Orthop. 1995; 321:106-10.

3. Ritter MA, Faris PM, Keating EM, Meding JB. Postoperative alignment of total knee replacement. Its effect on survival. Clin Orthop. 1994; 299:153-6.

4. Bäthis H, Perlick L, Tingart M, Luring C, Perlick C, Grifka J. Radiological results of image-based and non-image-based computer-assisted total knee arthroplasty. Int Orthop. 2004; 28: 87-90

5. Ecker ML, Lotke PA, Windsor RE, Cella JP. Long-term results after total condylar knee arthroplasty. Significance of radiolucent lines. Clin Orthop. 1987; 216:151-8.

6. Wasielewski RC, Galante JO, Leighty R, Natarajan RN, Rosenberg AG. Wear patterns on retrieved polyethylene tibial inserts and their relationship to technical considerations during total knee arthroplasty. Clin Orthop. 1994; 299:31-43.

7. Jeffery RS, Morris RW, Denham RA. Coronal alignment after total knee replacement. J Bone Joint Surg Br. 1991; 73:709-14.

8. Oswald MH, Jakob RP, Schneider E, Hoogewoud H. Radiological analysis of normal axial alignment of femur and tibia in view of total knee arthroplasty. $J$ Arthroplasty. 1993; 8:419-26.

9. Stulberg SD, Loan P, Sarin V. Computer-assisted navigation in total knee replacement: results of an initial experience in thirty-five patients. J Bone Joint Surg Am. 2002; 84(Suppl 2):90-8.

10. Thoma W., Schreiber S., Hovy L. Computer-assisted implant positioning in knee endoprosthetics. Kinematic analysis for optimization of surgical technique. Orthopade. 2000; 7:614-26

11. Jenny JY, Boeri C. Computer-assisted implantation of total knee prostheses: a case-control comparative study with classical instrumentation. Comput Aided Surg. 2001; 6:217-20

12. Sparmann M, Wolke B, Czupalla H, Banzer D, Zink A. Positioning of total knee arthroplasty with and without navigation support. A prospective, randomised study. J Bone Joint Surg Br. 2003; 85:830-5.
13. David Stulberg S. How accurate is current TKR instrumentation? Clin Orthop 2003; 416: 177-84.

14. Bathis H, Perlick L, Tingart M, Luring C, Zurakowski D, Grifka J. Alignment in total knee arthroplasty. A comparison of computer-assisted surgery with the conventional technique. J Bone Joint Surg Br. 2004; 86: 682-7.

15. Mihalko WM, Krackow KA. Differences between extramedullary, intramedullary, and computer-aided surgery tibial alignment techniques for total knee arthroplasty. J Knee Surg. 2006; 19: 33-6.

16. Zorman D, Etuin $P$, Jennart $H$, Scipioni D, Devos S. Computer-assisted total knee arthroplasty: comparative results in a preliminary series of 72 cases. Acta Orthop Belg. 2005; 71: 696-702.

17. Jenny JY, Clemens U, Kohler S, Kiefer H, Konermann W, Miehlke RK. Consistency of implantation of a total knee arthroplasty with a non-image-based navigation system a case-control study of 235 cases compared with 235 conventionally implanted prostheses. J Arthroplasty. 2005; 20: 832-9.

18. Kalairajah Y, Cossey AJ, Verrall GM, Ludbrook G, Spriggins AJ. Are systemic emboli reduced in computer-assisted knee surgery?: a prospective, randomised, clinical trial. J Bone Joint Surg Br. 2006; 88: 198-202

19. Kalairajah Y Simpson D, Cossey AJ, Verrall GM, Spriggins AJ. Blood loss after total knee replacement: effects of computer-assisted surgery. J Bone Joint Surg Br. 2005; 87: 1480-2.

20. Mielke RK, Clemens U, Jens JH, Kershally S. Navigation in knee endoprosthesis implantation--preliminary experiences and prospective comparative study with conventional implantation technique. Z Orthop Ihre Grenzgeb. 2001; 2:109-16

21. Clemens $U$, Miehlke RK. Advanced navigation planning including soft tissue management. Orthopedics. 2005; 28:1259-62.

22. Seon JK, Song EK. Functional impact of navigation-assisted minimally invasive total knee arthroplasty. Orthopedics. 2005; 28(10 Suppl):S1251-4.

23. Luring C, Hufner T, Perlick L, Bathis H, Krettek C, Grifka J. [Soft tissue management in knees with varus deformity Computer-assisted sequential medial ligament release.] Orthopade. 2005; 34(11):1118, 1120-2, 1124. 